\title{
The Emerging Role of Novel Therapies for the Treatment of Relapsed Myeloma
}

\author{
Paul G. Richardson, MD; ${ }^{\mathrm{a}}$ Teru Hideshima, MD, PhD; ${ }^{\mathrm{b}}$ Constantine Mitsiades, MD, PhD; and \\ Kenneth C. Anderson, MD, ${ }^{c}$ Boston, Massachusetts
}

\section{Key Words}

Bortezomib, proteasome inhibitor, thalidomide, immunomodulatory drug (IMiD), lenalidomide, multiple myeloma, relapsed

\begin{abstract}
Despite advances in the first-line treatment of multiple myeloma, almost all patients eventually relapse, become chemoresistant, and die of the disease. Improved understanding of potential myeloma targets and molecular mechanisms of drug resistance, along with the development and clinical investigation of targeted antitumor agents, have led to new strategies for the treatment of relapsed myeloma. The proteasome inhibitor bortezomib, the immunomodulatory agent thalidomide, and the thalidomide derivative lenalidomide, are all recently approved treatment options for myeloma. Single-agent bortezomib has been shown to provide significantly greater efficacy than high-dose dexamethasone, and bortezomib has also been investigated in combination with other agents commonly used to treat myeloma, including thalidomide and lenalidomide, with high overall and complete response rates. The safety profile of bortezomib has been well characterized, and side effects have been shown to be generally predictable and manageable, including in high-risk and elderly patients and those with renal impairment. Thalidomide has been extensively studied alone and in combination in patients with relapsed myeloma, demonstrating substantial efficacy, and is therefore widely used in this setting. The toxicity profile is doseand duration-linked, with lower doses appearing to be better tol-
\end{abstract}

\footnotetext{
From the Jerome Lipper Multiple Myeloma Center, Department of Adult Oncology, Dana-Farber Cancer Institute, and Harvard Medical School; ' Harvard Medical School, Dana-Farber Cancer Institute, and Brigham and Women's Hospital; and 'Jerome Lipper Multiple Myeloma Center, Kraft Family Donor Center, and Harvard Medical School, Boston, Massachusetts.

Submitted October 26, 2006; accepted for publication December 21, 2006

This paper was supported in part by grants: NIH SPORE IP50 CA10070-01, P0-1 78378, and R0-1 50947; the Doris Duke Distinguished Clinical Research Scientist Award; and the Cure for Myeloma Research Fund. Dr. Richardson receives research support from and is a consultant to Millennium Pharmaceuticals and Celgene; Dr. Anderson receives research support from and is a consultant to Millennium Pharmaceuticals, Celgene, and Novartis. Correspondence: Paul G. Richardson, MD, Dana-Farber Cancer Institute, 44 Binney Street, Dana 1B02, Boston, MA 02115. E-mail: paul_richardson@dfci.harvard.edu
}

erated. Lenalidomide plus dexamethasone has been shown to have significantly greater activity than dexamethasone alone in the relapsed setting, with impressive duration of disease control. Other combinations are also under investigation, with promising early results. Some aspects of the toxicity profile appear significantly reduced relative to thalidomide, although myelosuppression is increased. Other novel therapies at earlier stages of development are being studied and may provide further options in the treatment of relapsed myeloma. This review focuses on results from key phase II and III trials of bortezomib, thalidomide, and lenalidomide alone or in combination, and their emerging role in improving outcomes. (JNCCN 2007;5:149-162)

Despite advances in the first-line treatment of multiple myeloma (MM), including the introduction and widespread adoption of stem cell transplantation in patients younger than 70 years, ${ }^{1}$ the disease remains incurable. Almost all patients eventually experience relapse and develop drug-resistant disease. The pathophysiology of MM is intricate, involving many pathways and interactions between cytokines, adhesion molecules, mediators of angiogenesis, and signal transduction pathways. These elements taken together confer complex mechanisms of resistance while also providing multiple targets for novel therapeutic modalities. These targets are being exploited by a wide range of agents currently approved or under investigation, including bortezomib (Velcade, Millennium Pharmaceuticals, Cambridge, MA and Johnson \& Johnson Pharmaceutical Research and Development, Raritan, NJ), thalidomide (Thalomid, Celgene Corporation, Summit, NJ), and the thalidomide analogue lenalidomide (Revlimid, Celgene).

Single-agent bortezomib and lenalidomide plus dexamethasone are approved treatments for patients with relapsed MM who have undergone at least one previous therapy, and thalidomide plus dexamethasone is approved for the first-line treatment of MM. These novel therapeutic agents have substantially changed the treatment 
Richardson et al.

of MM and are widely used in the relapsed setting. However, the ultimate failure of therapy and the complex pathogenesis of this disease provide a rationale for further investigation of these agents, predominantly in combination, to determine their optimal use in the management of relapsed MM.

\section{Criteria of Response to Treatment}

Readers should note that trials investigating treatment for relapsed and for relapsed, refractory MM have used different systems for classifying patients' response to therapy. The Southwest Oncology Group (SWOG) and Eastern Cooperative Oncology Group have different standard sets of response criteria for their clinical trials, and different criteria again are used by the Intergroupe Francophone du Myelome in France and Medical Research Council in the UK. The stringent European Group for Blood and Marrow Transplantation (EBMT) criteria ${ }^{2}$ are currently considered a standard in clinical trials. These criteria result in reduced response rates compared with some earlier criteria.

Recognizing the need for uniform response criteria for MM, the International Myeloma Working Group recently published its International Response Criteria, updating the EBMT criteria. ${ }^{3}$ These are expected to become widely used in future clinical trials; in the meantime, comparisons of response rates and time to event data between trials should only be undertaken with due consideration for the response criteria used. The response rates reported herein are based on the EBMT criteria, unless otherwise stated.

\section{Bortezomib}

Bortezomib is the first approved agent in a new class of anticancer drugs called proteasome inhibitors, which block the ubiquitin-proteasome pathway. This pathway is responsible for the degradation of intracellular pro- teins; thus, blocking the proteasome results in disruption of protein homeostasis within the cell and dysregulation of cell cycle progression. Bortezomib has been shown to have multiple effects on molecular regulatory pathways within MM cells, causing changes in stress response, increased apoptotic susceptibility, and growth arrest (Figure 1, left). Furthermore, bortezomib affects the host environment by the inhibition of cytokine circuits, inhibition of angiogenesis in the bone marrow, and inhibition of MM cell adhesion (Figure 1 , right). ${ }^{4-7}$

MM is a particularly attractive target for proteasome inhibition because of the impact of the transcription factors nuclear factor (NF)- $\kappa B$ and interleukin (IL)-6, both indirectly regulated by proteasomal degradation, on the proliferation and survival of MM cells (Figure 1). Both indirect inhibition of NF- $\mathrm{KB}$ activity and activation of caspases by
MM Cellular Pathways

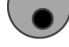
Apoptosis NF- $\mathrm{KB} \perp$ $\mathrm{BCl}-2 \downarrow, \mathrm{Bax} \uparrow$ p53 $\uparrow, \mathrm{mdm} 2 \downarrow$ JNK/SAPK activated Caspases 8, 9 activated TNF and TRAIL sensitization $\uparrow$ gp130 cleavage ICAM- $1 \downarrow$, VCAM- $1 \downarrow$

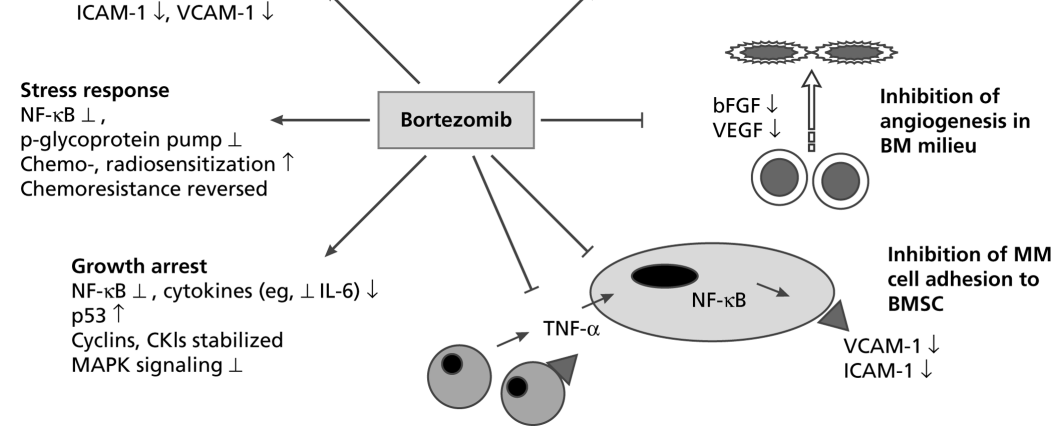

Figure 1 Effect of bortezomib on the MM cell and host microenvironment. Bortezomib inhibition of NF- $\mathrm{kB}$ and its effects on downstream targets and alternative pathways (Bcl-2Bax-Bad, p53/mdm2, JNK, caspases 8 and 9, sensitization to TNF/TRAIL-mediated apoptosis, and gp130 cleavage) causes changes in the susceptibility to apoptosis and survival mediated by cell adhesion pathways ICAM-1 and VCAM-1. NF- $\mathrm{kB}$ inhibition increases chemo- and radiosensitization and reverses chemoresistance. Affected cell signaling pathways include cyclins and CKIs, MAPK pathways, and cytokine circuits. Bortezomib directly and indirectly affects the host microenvironment by blocking the secretion of growth, survival, and migratory factors (IL-6, IGF-1, SDF- $1 \alpha$, VEGF, bFGF, TNF- $\alpha$ and TGF- $\beta$ ), angiogenic factors (VEGF and bFGF), and cell adhesion molecules (ICAM-1 and VCAM-1). Legand: $-\downarrow$ and $\perp=$ inhibition; $\rightarrow$ and $\uparrow=$ stimulation; $\downarrow=$ downregulation. Abbreviations: bFGF, basic fibroblast growth factor; BM, bone marrow; BMSC, bone marrow stromal cells; CKI, cyclin-dependent kinase inhibitor; ICAM, intracellular adhesion molecule; IGF, insulin-like growth factor; IL, interleukin; JNK, c-Jun-N-terminal kinase;

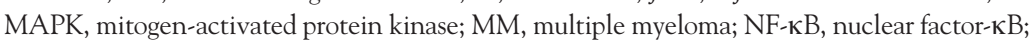
SAPK, stress-activated protein kinase; SDF, stromal-derived factor; TGF, transforming growth factor; TNF, tumor necrosis factor; TRAIL, TNF-related apoptosis-inducing ligand; VCAM, vascular cell adhesion molecule; VEGF, vascular endothelial growth factor. 
bortezomib trigger interruption of IL- 6 and IL-6 signaling cascades, respectively, which significantly inhibit the anti-apoptotic effects of IL-6 in MM cells. ${ }^{8}$ Other sequelae from the blockade of NF- $\mathrm{KB}$ include downregulation of antiapoptotic proteins such as $\mathrm{Bcl}$ 2 , upregulation of proapoptotic proteins such as Bax, and inhibition of adhesion molecules such as intracellular adhesion molecule 1 and vascular cell adhesion molecule (VCAM)-1. ${ }^{6}$ Furthermore, bortezomib leads to activation of both caspase 8- and 9-dependent apoptotic pathways, induction of p53 and c-Jun-N-terminal kinase in myeloma cells, and sensitization to tumor necrosis factor (TNF) and TNF-related apoptosis-inducing ligand (TRAIL)-dependent apoptosis., ${ }^{4,9-11}$

Other important pathways in the growth regulation of myeloma cells known to be influenced by proteasome inhibition are stabilization of cyclins and cyclin-dependent kinase inhibitors and inhibition of signaling through the p44/42 mitogen-activated protein kinase pathways., ${ }^{4,9,11}$ Additional studies suggest that modulation of proteasome function could sensitize cells to chemotherapy and reverse chemoresistance. ${ }^{12-14}$ Supporting the possibility of chemosensitization, studies have shown that bortezomib-based regimens can result in enhanced antitumor activity in models of MM with a variety of agents, including steroids, alkylating agents, anthracyclines, and immunomodulatory analogues of thalidomide. ${ }^{4,12,14,15}$

\section{Single-Agent Bortezomib}

The activity of single-agent bortezomib in patients with relapsed or relapsed, refractory MM was initially shown in 2 phase II studies, in which response rates between $27 \%$ and $38 \%$ were reported. ${ }^{16,17}$ Single-agent bortezomib was subsequently compared with high-dose dexamethasone in the international Assessment of Proteasome Inhibition for Extending Remissions (APEX) phase III trial of 669 patients with MM who had relapsed after 1 to 3 previous therapies. Initial analysis showed that bortezomib produced significantly greater median time to progression, response rate, and overall survival compared with dexamethasone. ${ }^{18}$ As a result, the dexamethasone arm was halted based on the recommendation of the independent data-monitoring committee, and patients on the dexamethasone arm were allowed to cross over to receive single-agent bortezomib.

In an updated analysis with extended follow-up (median 22 months), bortezomib again showed a significant survival advantage compared with dexa- methasone ( 29.8 vs. 23.7 months). This 6 -month benefit was observed despite more than $62 \%$ of dexamethasone-treated patients having crossed over to subsequently receive bortezomib. ${ }^{19}$ Indeed, bortezomib is the only single agent to date to show a survival benefit in this setting.

Updated efficacy data for the bortezomib arm also showed that response and complete or near complete response rates were $43 \%$ and $15 \%$, respectively (Table 1). ${ }^{19}$ The response rate and quality of responses continually improved over the course of protocol-specified treatment, after rapid initial response. In addition, greater depth of response (100\% M-protein reduction) was associated with longer duration of response. These data support extending treatment with bortezomib beyond initial response to achieve optimal benefit. ${ }^{19}$

Results from a number of subgroup analyses of the APEX study have been reported. An analysis in patients who had received 1 or more previous lines of therapy showed that bortezomib retained greater efficacy than dexamethasone in both groups of patients. The data also indicated that bortezomib has greater activity when used earlier in the relapsed setting. ${ }^{39}$ Furthermore, in a subgroup analysis to evaluate the impact of exposure to prior therapies on treatment outcome, bortezomib was consistently superior to dexamethasone regardless of types of prior therapies, including prior stem cell transplantation. ${ }^{40} \mathrm{~A}$ subgroup analysis has also assessed efficacy among elderly patients (aged $\geq 65$ years) and patients with other highrisk factors (including elevated $\beta_{2}$-microglobulin and insensitivity to prior therapy). Bortezomib retained its statistically superior efficacy compared with dexamethasone, in terms of longer median time to progression and higher response rate, in all patient subgroups studied. ${ }^{41}$

Finally, an analysis is being undertaken measuring the efficacy of bortezomib in patients with renal dysfunction in the APEX study. Renal dysfunction or failure is seen in up to $30 \%$ of MM patients at diagnosis. ${ }^{42,43}$ Studies in renally impaired patients, including those on dialysis, have shown that bortezomib-based therapy is feasible and shows encouraging activity, with similar toxicity to that seen in non-renally impaired patients, and rapid normalization of renal function in some. ${ }^{44-48}$

The most common toxicities seen with bortezomib treatment in the APEX trial included gastrointestinal events, fatigue, and peripheral neuropathy, with the 
Richardson et al.

\section{Table 1 Selected Clinical Trials of Bortezomib, Thalidomide, and Lenalidomide, Alone and In Combination, In the Treatment of Relapsed Multiple Myeloma

\begin{tabular}{|c|c|c|c|c|c|c|}
\hline Citation & Study & Regimen & $\begin{array}{l}\text { Patients } \\
\text { Enrolled } \\
(N)\end{array}$ & $C R+P R$ & $\begin{array}{l}\text { Respons } \\
\text { Rate } \\
(\%)^{*} \\
\text { CR/nCR }\end{array}$ & $\begin{array}{l}\text { Comparative } \\
\text { Major Toxicities }\end{array}$ \\
\hline
\end{tabular}

Phase 3 studies of bortezomib, and lenalidomide + dexamethasone (no phase III studies of thalidomide in the relapsed setting)

Richardson Single-agent bortezomib

et al. ${ }^{18,19} \quad$ vs.

high-dose dexamethasone

$1,4,8$, and 11 for up to

83 -week cycles followed

by treatment on days

$1,8,15$, and 22 for up to

35 -week cycles

Dexamethasone $40 \mathrm{mg}$ on days $1-4,9-12$, and $17-20$ for 45 -week cycles, followed by treatment on days 1-4 for 5 4-week cycles

Weber et al. ${ }^{20 \dagger}$ Lenalidomide + dexamethasone vs. dexamethasone

Dimopoulos et al. ${ }^{21 \neq}$

Lenalidomide + dexamethasone vs dexamethasone

Dexamethasone as above
Lenalidomide $25 \mathrm{mg}$ on days

1-21

Dexamethasone $40 \mathrm{mg}$

on days $1-4,9-12,17-20$

(days 1-4 only from cycle 5)

4-week cycles

170

2

Lenalidomide $25 \mathrm{mg}$ on days

176

1-21

Dexamethasone $40 \mathrm{mg}$ on days

$1-4,9-12,17-20$ (days $1-4$

only from cycle 5)

4-week cycles

Dexamethasone as above

175

171
333

43

15

15

Grade 3/4:

thrombocytope-

nia $26 / 4 \%$,

neutropenia

12/2\%; anemia

$9 / 1 \%$, peripheral

neuropathy

$7 / 1 \%$

336

18

2

Grade 3/4:

anemia $10 / 1 \%$, thrombocytopenia $5 / 1 \%$

59

13

Grade 3/4:

neutropenia

$36 \%$,

thrombocytope-

nia $12 \%$, periph-

eral neuropathy

$12 \%$, anemia

$12 \%$, pneumo-

nia $>10 \%$

DVT/PE 18\%

Grade 3/4: thrombocytopenia $6 \%$, neutropenia $5 \%$, anemia $5 \%$, fatigue $5 \%$

Grade 3/4: neutropenia $27 \%$, thrombocytopenia $10 \%$, anemia $8 \%$, DVT $5 \%$, PE 4\%

Grade 3/4: thrombocytopenia $6 \%$, DVT $5 \%$

Selected phase I and II studies of bortezomib, thalidomide, and lenalidomide

\begin{tabular}{|c|c|c|c|c|c|c|}
\hline $\begin{array}{l}\text { Hollmig } \\
\text { et al. } 22 \|\end{array}$ & $\begin{array}{l}\text { Bortezomib }+ \\
\text { doxorubicin }+ \text { thalidomide } \\
+ \text { dexamethasone } \\
\text { (phase 1) }\end{array}$ & $\begin{array}{l}\text { Bortezomib } 0.8-1.3 \mathrm{mg} / \mathrm{m}^{2} \text { on } \\
\text { days } 1,4,8,11 \\
\text { Doxorubicin } 2.5-5 \mathrm{mg} / \mathrm{m}^{2} \\
\text { on days } 1-4 \text { and } 9-12 \\
\text { Thalidomide } 50-100 \mathrm{mg} / \text { day } \\
\text { on days } 1-12 \text { Dexamethasone } \sqrt{ } \\
20-40 \mathrm{mg} \text { on days } 1-4 \text { and } 9-12\end{array}$ & 20 & $63^{\S}$ & $25^{\S}$ & $\begin{array}{l}\text { Grade } 3 \\
\text { thrombocytope- } \\
\text { nia in } 40 \%\end{array}$ \\
\hline
\end{tabular}


Novel Therapies in Relapsed Myeloma

\begin{tabular}{|c|c|c|c|c|c|c|}
\hline Leoni et al. ${ }^{23^{*}}$ & $\begin{array}{l}\text { Bortezomib + liposomal } \\
\text { doxorubicin (Myocet) }+ \\
\text { thalidomide }+ \\
\text { dexamethasone (phase } 2 \text { ) }\end{array}$ & $\begin{array}{l}\text { Bortezomib } 1.0 \mathrm{mg} / \mathrm{m}^{2} \text { on } \\
\text { days } 1,4,8, \text { and } 11 \\
\text { Myocet } 50 \mathrm{mg} / \mathrm{m}^{2} \text { on day } 4 \\
\text { Thalidomide } 100 \mathrm{mg} / \text { day } \\
\text { Dexamethasone } 24 \mathrm{mg} \text { on day } \\
\text { of/day after bortezomib } \\
\text { Up to } 4 \text {-week cycles }\end{array}$ & 27 & 74 & 33 & $\begin{array}{l}\text { Grade } 4 \text { hemato- } \\
\text { logic toxicity in } \\
18 \%\end{array}$ \\
\hline $\begin{array}{l}\text { Zangari } \\
\text { et al.24ף }\end{array}$ & $\begin{array}{l}\text { Bortezomib }+ \text { thalidomide } \\
\pm \text { dexamethasone (phase } 2 \text { ) }\end{array}$ & $\begin{array}{l}\text { Bortezomib } 1.0-1.3 \mathrm{mg} / \mathrm{m}^{2} \text { on } \\
\text { days } 1,4,8,11 \\
\text { Thalidomide } 50-20 \mathrm{mg} / \text { day } \\
\text { from cycle } 2 \\
\text { Dexamethasone } 20 \mathrm{mg} \text { on day } \\
\text { of/day after bortezomib for } \\
\text { suboptimal response after } \\
3 \text { cycles } \\
\text { Up to } 8 \text { 3-week cycles }\end{array}$ & 85 & $55^{\S}$ & $16^{\S}$ & $\begin{array}{l}\text { Most common } \\
\text { grade } 3 / 4 \text { : } \\
\text { thrombocy- } \\
\text { topenia and } \\
\text { neutropenia }\end{array}$ \\
\hline $\begin{array}{l}\text { Orlowski } \\
\text { et al. }{ }^{25}\end{array}$ & $\begin{array}{l}\text { Bortezomib + pegylated } \\
\text { liposomal doxorubicin } \\
\text { (phase 1) }\end{array}$ & $\begin{array}{l}\text { Bortezomib } 0.9-1.5 \mathrm{mg} / \mathrm{m}^{2} \text { on } \\
\text { days } 1,4,8 \text {, and } 11 \\
\text { Liposomal doxorubicin } \\
30 \mathrm{mg} / \mathrm{m}^{2} \text { on day } 4 \\
\text { Up to } 11 \text { 3-week cycles }\end{array}$ & 24 & 73 & 36 & $\begin{array}{l}\text { Grade } 3 / 4 \text { in all } \\
42 \text { patients } \\
\text { with hematologic } \\
\text { malignan- } \\
\text { cies: thrombocy- } \\
\text { topenia } 43 \% \text {, } \\
\text { lymphopenia } \\
40 \% \text {, neutropenia } \\
17 \% \text {, fatigue } \\
14 \% \text {, pneumonia } \\
14 \% \text {, peripheral } \\
\text { neuropathy } \\
12 \% \text {, febrile } \\
\text { neutropenia } \\
10 \% \text {, diarrhea } \\
10 \%\end{array}$ \\
\hline $\begin{array}{l}\text { Richardson } \\
\text { et al. }{ }^{26 \uparrow}\end{array}$ & $\begin{array}{l}\text { Bortezomib + lenalidomide } \\
\pm \text { dexamethasone } \\
\text { (phase 1) }\end{array}$ & $\begin{array}{l}\text { Bortezomib } 1.0-1.3 \mathrm{mg} / \mathrm{m}^{2} \\
\text { on days } 1,4,8,11 \\
\text { Lenalidomide } 5-20 \mathrm{mg} \text { on } \\
\text { days } 1-14 \\
\text { Dexamethasone } 20 \mathrm{mg} \text { on } \\
\text { day of/day after bortezomib } \\
\text { for PD } \\
\text { Up to } 8 \text { 3-week cycles }\end{array}$ & 24 & 52 & 10 & $\begin{array}{l}\text { Grade 3/4: } \\
\text { thrombocytope- } \\
\text { nia, neutropenia, } \\
\text { and hypon } \\
\text { atremia. No } \\
\text { significant fa- } \\
\text { tigue or periph- } \\
\text { eral neuropathy }\end{array}$ \\
\hline $\begin{array}{l}\text { Padmanabhan } \\
\text { et al. } 27 \neq\end{array}$ & $\begin{array}{l}\text { Bortezomib + liposomal } \\
\text { doxorubicin + thalidomide } \\
\text { (phase } 2 \text { ) }\end{array}$ & $\begin{array}{l}\text { Bortezomib } 1.3 \mathrm{mg} / \mathrm{m}^{2} \text { on days } \\
1,4,15 \text {, and } 18 \\
\text { Liposomal doxorubicin } \\
20 \mathrm{mg} / \mathrm{m}^{2} \text { on days } 1 \text { and } 15 \\
\text { Thalidomide } 200 \mathrm{mg} / \mathrm{day} \\
\text { Up to } 64 \text {-week cycles }\end{array}$ & 23 & $65^{* *}$ & $23^{* *}$ & $\begin{array}{l}\text { No grade } 3 / 4 \\
\text { non-hematologic } \\
\text { toxicities }\end{array}$ \\
\hline $\begin{array}{l}\text { Palumbo } \\
\text { et al. }{ }^{28 \neq}\end{array}$ & $\begin{array}{l}\text { Bortezomib }+ \text { melphalan }+ \\
\text { prednisone }+ \text { thalidomide } \\
\text { (phase } 2)\end{array}$ & $\begin{array}{l}\text { Bortezomib } 1-1.6 \mathrm{mg} / \mathrm{m}^{2} \text { on } \\
\text { days } 1,4,15,22 \\
\text { Melphalan } 6 \mathrm{mg} / \mathrm{m}^{2} \text { and } \\
\text { prednisone } 60 \mathrm{mg} / \mathrm{m}^{2} \text { on } \\
\text { days } 1-5 \\
\text { Thalidomide } 50 \mathrm{mg} \\
\text { continuously } \\
65 \text {-week cycles }\end{array}$ & 30 & 67 & 17 & $\begin{array}{l}\text { Grade 3/4: } \\
\text { thrombocytopenia, } \\
\text { neutropenia, } \\
\text { fatigue, anemia, } \\
\text { vasculitis, } \\
\text { infections, and } \\
\text { sensory } \\
\text { neuropathy }\end{array}$ \\
\hline
\end{tabular}


Richardson et al.

\begin{tabular}{|c|c|c|c|c|c|c|}
\hline Terpos et al. ${ }^{29 \ddagger}$ & $\begin{array}{l}\text { Bortezomib + melphalan }+ \\
\text { dexamethasone }+ \\
\text { thalidomide (phase 2) }\end{array}$ & $\begin{array}{l}\text { Bortezomib } 1.0 \mathrm{mg} / \mathrm{m}^{2} \text { on } \\
\text { days } 1,4,8 \text {, and } 11 \\
\text { Melphalan } 0.15 \mathrm{mg} / \mathrm{kg} \text { days } \\
1-4 \\
\text { Dexamethasone } \\
12 \mathrm{mg} / \mathrm{m}^{2} \text { days } 1-4 \text { and } 17-20 \\
\text { Thalidomide } 100 \mathrm{mg} / \text { day } \\
\text { Up to } 84 \text {-week cycles }\end{array}$ & 44 & 66 & 37 & $\begin{array}{l}\text { Grade } 3 / 4 \text { : } \\
\text { thrombocytope- } \\
\text { nia } 20 \% \text {, neu- } \\
\text { tropenia } 8 \% \text {, } \\
\text { anemia } 7 \% \text {, and } \\
\text { peripheral neu- } \\
\text { ropathy } 6 \%\end{array}$ \\
\hline $\begin{array}{l}\text { Barlogie } \\
\text { al. }{ }^{30}\end{array}$ & Thalidomide (phase 2) & Thalidomide $200-800 \mathrm{mg} /$ day & 169 & $30^{\S}$ & $14^{\S}$ & $\begin{array}{l}\text { Grade } 3 / 4 \text { : CNS } \\
25 \% \text {, gastroin- } \\
\text { testinal } 16 \% \text {, } \\
\text { peripheral } \\
\text { neuropathy } 9 \%\end{array}$ \\
\hline $\begin{array}{l}\text { Palumbo } \\
\text { et al. }{ }^{31}\end{array}$ & $\begin{array}{l}\text { Thalidomide }+ \\
\text { dexamethasone } \\
\text { (at first relapse) } \\
\text { (phase II) } \\
\text { Thalidomide + } \\
\text { dexamethasone } \\
\text { (at second relapse and } \\
\text { beyond) }\end{array}$ & $\begin{array}{l}\text { Thalidomide } 100 \mathrm{mg} / \text { day } \\
\text { continuously } \\
\text { Dexamethasone } 40 \mathrm{mg} \text { on } \\
\text { days } 1-4 \text { of each month }\end{array}$ & 62 & $56^{\S}$ & & $\begin{array}{l}\text { Grade } 3 / 4 \text { : } \\
\text { tingling and } \\
\text { numbness } 19 \% \text {, } \\
\text { constipation } 18 \% \text {, } \\
\text { sedation } 13 \%\end{array}$ \\
\hline $\begin{array}{l}\text { Dimopoulos } \\
\text { et al. }{ }^{32}\end{array}$ & $\begin{array}{l}\text { Thalidomide + } \\
\text { dexamethasone } \\
+ \text { pulsed } \\
\text { cyclophosphamide } \\
\text { (phase II) }\end{array}$ & $\begin{array}{l}\text { Thalidomide } 400 \mathrm{mg} / \text { day on } \\
\text { days } 1-5 \text { and } 14-18 \\
\text { Dexamethasone } 20 \mathrm{mg} / \mathrm{day} \\
\text { on days } 1-5 \text { and } 14-18 \\
\text { Cyclophosphamide } 150 \mathrm{mg} / \mathrm{m}^{2} \\
\text { every } 12 \text { hours on days } 1-5 \\
34 \text {-week cycles }\end{array}$ & 53 & $60^{\S}$ & $5^{\S}$ & $\begin{array}{l}\text { Grade } 3 / 4 \text { : neu- } \\
\text { tropenia } 18 / 8 \% \text {, } \\
\text { thrombocytope- } \\
\text { nia } 0 / 2 \%\end{array}$ \\
\hline $\begin{array}{l}\text { Offidani } \\
\text { et al. }{ }^{33}\end{array}$ & $\begin{array}{l}\text { Thalidomide }+ \\
\text { liposomal doxorubicin }+ \\
\text { dexamethasone (phase } 2 \text { ) }\end{array}$ & $\begin{array}{l}\text { Thalidomide } 100 \mathrm{mg} / \text { day } \\
\text { Liposomal doxorubicin } \\
40 \mathrm{mg} / \mathrm{m}^{2} \text { on day } 1 \\
\text { Dexamethasone } 40 \mathrm{mg} \text { on days } \\
1-4 \text { and } 9-12 \\
\text { 4-week cycles }\end{array}$ & 50 & 76 & 32 & $\begin{array}{l}\text { Grade } 3 / 4 \text { : } \\
\text { neutropenia } \\
16 \% \text {, severe } \\
\text { infection } 16 \% \text {, } \\
\text { plus venous } \\
\text { thromboembolic } \\
\text { disease } 12 \%\end{array}$ \\
\hline $\begin{array}{l}\text { Hussein } \\
\text { et al. }{ }^{34}\end{array}$ & $\begin{array}{l}\text { Thalidomide }+ \\
\text { liposomal doxorubicin }+ \\
\text { vincristine }+ \\
\text { dexamethasone (phase } 2 \text { ) }\end{array}$ & $\begin{array}{l}\text { Thalidomide } 50-400 \mathrm{mg} / \mathrm{day} \\
\text { Liposomal doxorubicin } \\
40 \mathrm{mg} / \mathrm{m}^{2} \text { on day } 1 \\
\text { Vincristine } 2 \mathrm{mg} \text { on day } 1 \\
\text { Dexamethasone } 40 \mathrm{mg} \text { on } \\
\text { days } 1-4 \\
>64 \text {-week cycles }\end{array}$ & 49 & 76 & 20 & $\begin{array}{l}\text { Grade } 3 / 4 \text { : neu- } \\
\text { ropathy } 22 \%, \\
\text { neutropenia } 14 \% \text {, } \\
\text { pneumonia } 12 \% \text {, } \\
\text { palmar plantar } \\
\text { erythrodysthesia } \\
8 \%, \text { fatigue } 6 \% \text {, } \\
\text { thrombocytope- } \\
\text { nia } 5 \% \text { (includes } \\
53 \text { patients with } \\
\text { newly diagnosed } \\
\text { disease) }\end{array}$ \\
\hline $\begin{array}{l}\text { Palumbo } \\
\text { et al. }{ }^{35}\end{array}$ & $\begin{array}{l}\text { Thalidomide + } \\
\text { melphalan }+ \\
\text { prednisone (phase 2) }\end{array}$ & $\begin{array}{l}\text { Thalidomide } 50-100 \mathrm{mg} / \mathrm{day} \\
\text { continuously } \\
\text { Prednisone } 50 \mathrm{mg} / \text { day every } \\
\text { other day } \\
\text { Intravenous melphalan } \\
20 \mathrm{mg} / \mathrm{m}^{2} \text { every fourth month }\end{array}$ & 24 & 42 & 13 & $\begin{array}{l}\text { Grade } 3 / 4 \text { : } \\
\text { constipation, } \\
\text { tingling, } \\
\text { and sedation }\end{array}$ \\
\hline $\begin{array}{l}\text { Richardson } \\
\text { et al. }{ }^{36 \pi}\end{array}$ & Lenalidomide (phase 2) & $\begin{array}{l}\text { Lenalidomide } 30 \mathrm{mg} \text { on } \\
\text { days } 1-21 \\
\text { 4-week cycles }\end{array}$ & 222 & 25 & & $\begin{array}{l}\text { Grade } 3 / 4 \text { : upper } \\
\text { respiratory } \\
\text { tract infection, } \\
\text { neutropenia, } \\
\text { thrombocytopenia }\end{array}$ \\
\hline
\end{tabular}


Novel Therapies in Relapsed Myeloma

\begin{tabular}{|c|c|c|c|c|c|c|}
\hline $\begin{array}{l}\text { Gerecke } \\
\text { et al. } 37 \text { ๆ }\end{array}$ & $\begin{array}{l}\text { Lenalidomide }+ \\
\text { doxorubicin }+ \\
\text { dexamethasone (phase 1) }\end{array}$ & $\begin{array}{l}\text { Lenalidomide } 10 / 15 \mathrm{mg} \text { on } \\
\text { days } 1-21 \\
\text { Doxorubicin } 4 / 6 / 9 \mathrm{mg} / \mathrm{m}^{2} \\
\text { on days } 1-4 \\
\text { Dexamethasone } 40 \mathrm{mg} \text { on days } \\
1-4 \text { and } 17-20 \\
3-6 \text {-week cycles }\end{array}$ & 6 & \multicolumn{2}{|c|}{$\begin{array}{l}\text { All patients } \\
\text { responded }\end{array}$} & $\begin{array}{l}\text { Well tolerated } \\
\text { at first } 2 \text { dose } \\
\text { levels }\end{array}$ \\
\hline Baz et al. ${ }^{38}$ & $\begin{array}{l}\text { Lenalidomide }+ \\
\text { liposomal doxorubicin }+ \\
\text { vincristine }+ \\
\text { dexamethasone } \\
\text { (phase } 2 \text { ) }\end{array}$ & $\begin{array}{l}\text { Lenalidomide } \leq \\
\leq 10 \mathrm{mg} \text { on days } 1-21 \\
\text { Liposomal doxorubicin } \\
40 \mathrm{mg} / \mathrm{m}^{2} \text { on day } 1 \\
\text { Vincristine } 2 \mathrm{mg} \text { on day } 1 \\
\text { Dexamethasone } 40 \mathrm{mg} \text { on } \\
\text { days } 1-4 \text {-week cycles }\end{array}$ & 62 & $75^{* *}$ & $29 * *$ & $\begin{array}{l}\text { Grade } 3 / 4 \text { : neu- } \\
\text { tropenia } 32 \% \text {, } \\
\text { thrombocyto- } \\
\text { penia } 13 \% \text {, } \\
\text { infectious } 13 \% \text {, } \\
\text { DVT/PE } 9 \%\end{array}$ \\
\hline
\end{tabular}

*EBMT criteria unless otherwise stated.

${ }^{\dagger}$ Data presented at the 2006 Annual Meeting of the American Society of Clinical Oncology.

${ }^{\ddagger}$ Data presented at the 2006 meeting of the European Hematology Association.

IIData presented at the 2004 Annual Meeting of the American Society of Hematology.

$\S \mathrm{M}$-protein reduction.

IData presented at the 2005 Annual Meeting of the American Society of Hematology.

${ }^{* *}$ SWOG criteria.

Abbreviations: CNS, central nervous system; CR, complete response; DVT, deep vein thrombosis; $n C R$, near complete response; PD, progressive disease; PE, pulmonary embolism; PR, partial response.

most common grade 3 or 4 bortezomib-associated toxicities being thrombocytopenia, neutropenia, anemia, and peripheral neuropathy. ${ }^{18}$ Grade 3 or 4 adverse events were more common with bortezomib than with high-dose dexamethasone; however, a substantial proportion of these events in the bortezomib arm were thrombocytopenia or neutropenia, which have been shown to be transient and cyclical. ${ }^{49,50}$ For example, in patients with thrombocytopenia, platelet counts decrease during each cycle of bortezomib dosing and then recover predictably during the rest period, with no evidence of cumulative toxicity. ${ }^{49}$ Notably, despite a higher incidence of grade 3 or 4 thrombocytopenia in the bortezomib arm compared with the dexamethasone arm, the incidence of significant bleeding events was similar between arms. ${ }^{49}$ Bortezomib-associated peripheral neuropathy has been shown to be reversible in most patients. ${ }^{51,52}$ In the APEX study, $27 \%$ of patients developed grade 2 or higher peripheral neuropathy. Of these patients, the neuropathy resolved or improved in approximately two-thirds: $55 \%$ experienced complete return to baseline and $9 \%$ improved by at least 1 grade. ${ }^{52}$

\section{Bortezomib-Based Combinations}

Bortezomib has been extensively investigated in combination with other agents commonly used to treat MM, including thalidomide and lenalidomide (Table
1). Ongoing phase III studies, from which results have yet to be reported, are investigating the combination of bortezomib plus thalidomide and dexamethasone (VTD regimen) versus thalidomide and dexamethasone or VTD plus doxorubicin (VATD regimen). Both the VTD and VATD regimens have been shown to be active in phase II studies in patients with relapsed or relapsed, refractory MM. ${ }^{22-24}$ A response rate (Mprotein reduction) of $55 \%$, including $16 \%$ complete or near complete responses, was reported in 1 study of VTD, along with median event-free and overall survival times of 9 and 22 months, respectively. ${ }^{24}$ VATD has been shown to produce a $63 \%$ response rate, with $25 \%$ complete or near complete responses (M-protein reduction), ${ }^{22}$ and recently reported data show that the addition of liposomal doxorubicin to the VTD regimen can increase the response rate to $74 \%$, with $33 \%$ complete or near complete responses. ${ }^{23}$

Other combinations that have produced high response rates (up to $73 \%$ ) $^{25}$ and complete or near complete response rates (up to $36 \%$ ) ) $^{25}$ in the relapsed setting include bortezomib plus liposomal doxorubicin alone ${ }^{37}$ or with thalidomide, ${ }^{27}$ lenalidomide, ${ }^{26}$ and melphalan plus thalidomide, with or without steroids. ${ }^{28,29}$ The safety profile in these combination studies has been as expected, with no additive toxicities reported, and, in fact, better tolerability described. Bortezomib can thus be combined safely and effectively with a 
Richardson et al.

broad range of both commonly used and novel MM agents.

\section{Thalidomide}

Multiple studies have shown that thalidomide has various effects on myeloma cell growth and the host microenvironment. ${ }^{53}$ Given its broad spectrum of therapeutic activity, thalidomide may have several possible mechanisms of action in MM cells (Figure 2). ${ }^{54}$ First, it may inhibit myeloma or bone marrow stromal cell growth and survival while concurrently activating pro-apoptotic pathways. ${ }^{55}$ Second, thalidomide may inhibit vascular endothelial growth factor and basic fibroblast growth factor activity and angiogen$\mathrm{esis}^{56}$ and modulate adhesion, thereby affecting tumor cell growth, survival, and drug resistance..$^{57}$ Third, thalidomide may alter the secretion and bioactivity of cytokines released into the bone marrow environment. ${ }^{55}$ Finally, its activity may in part be exerted via the immunomodulatory properties of the compound..$^{58}$ dose (29\%). ${ }^{64}$ Further studies to confirm this observation therefore seem to be warranted.

The toxicity profile of thalidomide appears both dose- and duration-linked. Drug-related adverse events associated with thalidomide treatment in heavily pretreated patients with refractory disease include neurologic (drowsiness, numbness, dizziness, confusion, tremor, lack of coordination, and tingling) gastrointestinal (constipation, stomatitis, nausea, and vomiting), and constitutional (weight loss, weakness, and fever) effects. ${ }^{65,66}$ Thromboembolic events reported during thalidomide therapy include deep vein thrombosis, pulmonary embolus, and clotting of central venous catheters. ${ }^{66}$ The risk of these events with thalidomide therapy may be attributed to the finding that thalidomide significantly increases thrombin generation in patients with $\mathrm{MM}$, as a measure of hypercoagulability. ${ }^{67}$

Peripheral neuropathy has been reported to affect between $1 \%$ and $50 \%$ of patients in clinical trials. ${ }^{66}$

\section{Thalidomide as a Single-Agent and in Combination}

No phase III studies have been reported on thalidomide in the relapsed setting, although a number are in progress. However, it has been extensively used either alone or in combination regimens in this setting (Table 1). Singleagent thalidomide has been shown to be effective in approximately one third of patients with relapsed or refractory MM in a number of phase II studies, ${ }^{30,59-62}$ and a recent systematic review of published clinical trials found the mean complete and partial response rate (>50\% reduction in M-protein) to be $29 \%$, with a median overall survival of 14 months. ${ }^{63}$ Most studies of thalidomide monotherapy have used a single daily dose administered in the evening to minimize the effect of sedation, as opposed to multiple divided dosing. One trial directly compared single- to multiple-dose thalidomide; patients receiving divided daily doses showed an improved response rate (Mprotein reduction) (50\%) compared with those who received a single daily

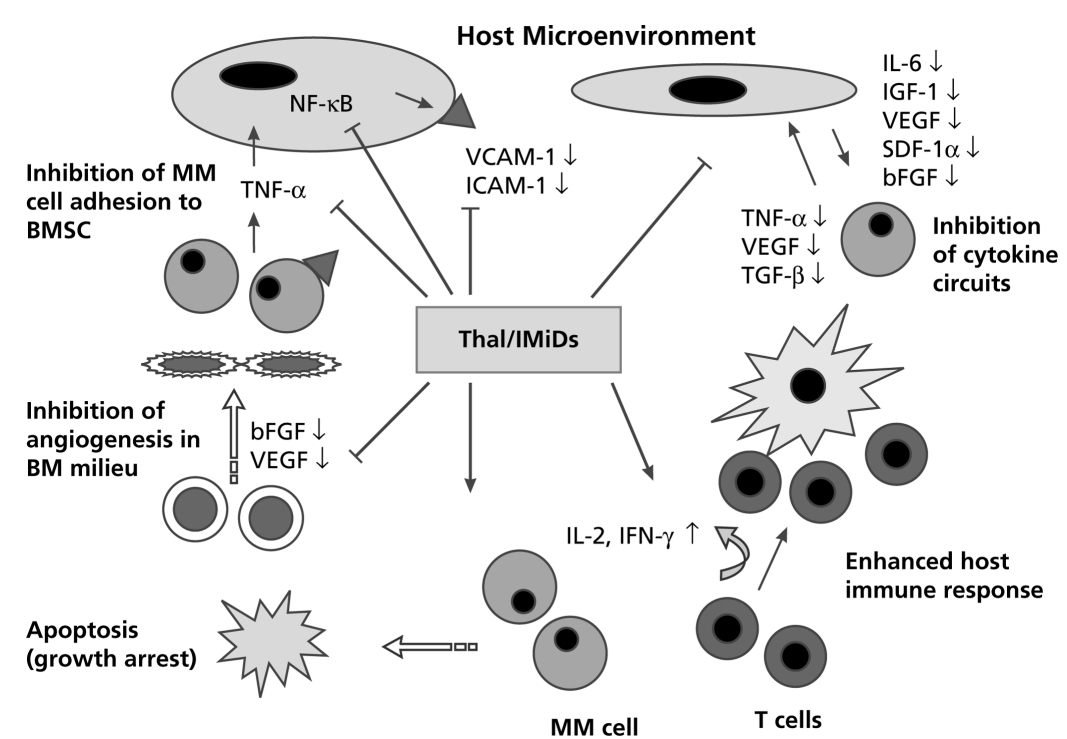

Figure 2 Effect of thalidomide (Thal) and immunomodulatory drugs (IMiDs) on the myeloma cell and its bone marrow microenvironment. Thalidomide and IMiDs affect multiple cellular pathways, causing MM cell apoptosis and growth arrest. These compounds directly and indirectly affect the host microenvironment by blocking the secretion of growth, survival, and migratory factors (IL-6, IGF-1, SDF-1 $\alpha$, VEGF, bFGF, TNF- $\alpha$ and TGF- $\beta$ ), angiogenic factors (VEGF and bFGF), and cell adhesion molecules (ICAM- 1 and VCAM-1). Thalidomide and IMiDs expand natural killer cell and T-cell numbers and enhance myeloma cell susceptibility to ADCC in vivo. Legend: $-\downarrow$ and $\perp=$ inhibition; $\rightarrow$ and $\uparrow=$ stimulation; $\downarrow=$ downregulation. Abbreviations: ADCC, antibody-dependent cell-mediated cytotoxicity; bFGF, basic fibroblast growth factor; BM, bone marrow; BMSC, bone marrow stromal cells; ICAM, intracellular adhesion molecule; IGF, insulin-like growth factor; IFN, interferon; IL, interleukin; MM, multiple myeloma; NF- $\kappa B$, nuclear factor $\kappa \mathrm{B}$; SDF, stromal-derived factor; TGF, transforming growth factor; TNF, tumor necrosis factor; VCAM, vascular cell adhesion molecule; VEGF, vascular endothelial growth factor. Modified from Richardson et al. ${ }^{54}$ with permission. 
Symptoms may improve when thalidomide is discontinued; however, recovery may not be total and symptoms may also progress after discontinuation of therapy ${ }^{66}$ In one retrospective analysis of a phase II study, researchers found that $56 \%$ of patients developed symptoms of peripheral neuropathy and suggested that the incidence and severity of peripheral neuropathy are likely related to dose and duration of therapy. ${ }^{68}$ In another study of thalidomide in patients with relapsed or refractory disease, $41 \%$ of patients and $81 \%$ of responding patients were seen to develop peripheral neuropathy, with the actuarial incidence among patients remaining on therapy increasing from 38\% at 6 months to $73 \%$ at 12 months; $15 \%$ of patients discontinued thalidomide because of this toxicity. ${ }^{69}$ Based on these results, the researchers recommended that thalidomide therapy should be limited to less than 6 months to minimize the risk of neurotoxicity ${ }^{69}$

Because of the dose-linked toxicity profile of thalidomide, lower doses than the median of 300 to $400 \mathrm{mg}$ used in most studies have been explored. A recent prospective study in 400 patients with relapsed or refractory MM has shown that a $100 \mathrm{mg}$ daily dose of thalidomide is comparable in terms of overall survival to a dose of $400 \mathrm{mg}$ daily and is better tolerated..$^{70}$

The clinical benefit of thalidomide increases when it is combined with other agents, although the elevated risk of deep vein thrombosis is an important safety consideration..$^{71,72}$ The risk increases substantially when thalidomide is combined with corticosteroid therapy ${ }^{73,74}$ or other therapeutic agents, such as anthracyclines. ${ }^{75}$ Prophylaxis with warfarin or low molecular weight heparin (LMWH) should be used to manage this risk. ${ }^{72,76}$ Although the use of low-dose aspirin may help reduce the incidence, ${ }^{75}$ researchers have suggested that aspirin should only be used in patients unable or unwilling to take warfarin or LMWH. ${ }^{76}$

Thalidomide in combination with dexamethasone therapy is a commonly-used first-line regimen for MM in the United States and has also shown efficacy in relapsed disease in a number of phase II studies. ${ }^{31,77-80}$ A recent retrospective analysis of studies of patients with relapsed or refractory MM reports that thalidomide plus dexamethasone may be superior to thalidomide alone in this group of patients, with response rates (M-protein reduction $>50 \%$ ) of $51 \%$ and $29 \%$, respectively. ${ }^{81}$ The addition of cyclophosphamide to thalidomide and dexamethasone (CTD) appears to further increase activity in the relapsed or refractory setting, with response rates (using various criteria) of up to $83 \%$ and complete response rates of up to $17 \%$ reported. ${ }^{32,82-84}$

The combination of thalidomide with liposomal doxorubicin and high-dose dexamethasone has also been investigated in a phase II study. ${ }^{33}$ The overall response rate was $76 \%$, and $32 \%$ of patients experienced a complete or near complete response. Median progression-free and event-free survival times were 22 and 17 months, respectively. ${ }^{33}$ This triplet regimen was compared with thalidomide plus dexamethasone in a case-matched study in patients with advanced $\mathrm{MM}$, and resulted in a higher response rate (76\% vs. $60 \%$ ), and rate of complete or near complete responses (30\% vs. $11 \%$ ). ${ }^{85}$ Median progressionfree survival was also significantly longer with the triplet combination (22 vs. 12 months). However, the incidence of vascular events was also greater with the triplet regimen (13\% vs. $6 \%){ }^{85}$

Thalidomide has also been combined with liposomal doxorubicin, vincristine, and dexamethasone (DVd-T). An overall response rate of $76 \%$ was seen, but toxicities included $25 \%$ grade 3 or 4 thromboembolic events and $22 \%$ grade 3 or 4 peripheral neuropathy. ${ }^{34}$ Finally, a small study investigating the combination of thalidomide, melphalan, and prednisone in patients with refractory and relapsed myeloma showed a response rate of $42 \%$, including $13 \%$ near complete responses, with acceptable levels of toxicity.

\section{Lenalidomide}

Thalidomide has shown great promise in the treatment of MM; however, its toxicity profile has spurred the development of thalidomide-derived analogues, also known as immunomodulatory drugs (IMiDs), with the potential of improved potency and reduced toxicity. Like thalidomide, the other IMiDs inhibit angiogenesis, induce both apoptosis and growth arrest in resistant myeloma cells, and inhibit myeloma cell adhesion to bone marrow stromal cells (Figure 2). ${ }^{53}$ Furthermore, this inhibition of cell adhesion leads to a blockade of myeloma cell growth, survival, and migratory factor secretion. These compounds expand natural killer cell and T-cell numbers and function against human myeloma cells and enhance their susceptibility to antibody-dependent cell-mediated 
Richardson et al.

cytotoxicity in vivo. ${ }^{53,55,86-89}$ Lenalidomide is the most advanced IMiD in terms of clinical development, and has been investigated in relapsed and first-line settings.

\section{Lenalidomide as a Single Agent and in Combination}

As with thalidomide, lenalidomide as a single agent is active in the relapsed setting, with significant responses seen in approximately one fourth of patients. ${ }^{36,90,91}$ However, again as seen with thalidomide, activity is greatly increased in combination with dexamethasone (Table 1). The combination regimen was compared with dexamethasone alone in 2 phase III trials in North America (MM-009) ${ }^{20}$ and Europe (MM010). ${ }^{21}$ In the North American study, a response rate of $59 \%$ was seen with lenalidomide plus dexamethasone, including $13 \%$ complete responses. This was significantly greater than the $21 \%$ response rate and $1 \%$ complete response rate seen with dexamethasone alone in this study. ${ }^{20}$ The combination regimen also resulted in a significantly longer median time to progression of 11.1 months compared with 4.7 months with dexamethasone, and an extended median survival of 29.6 versus 20.2 months with dexamethasone. ${ }^{20}$

A response rate of $59 \%$ was also seen with lenalidomide plus dexamethasone in the European phase III study, compared with $24 \%$ with dexamethasone. Complete response rates were 15\% and $3 \%$, respectively. ${ }^{21}$ Time-to-events data were also similar to those seen in the North American study; median time to progression was 11.3 months with the combination compared with 4.7 months with dexamethasone. After 27.9 months of follow up, median survival had not been reached in patients receiving lenalidomide plus dexamethasone, compared with 20.6 months in those receiving dexamethasone..$^{21}$

A pooled analysis of these 2 studies examined the efficacy of lenalidomide plus dexamethasone in patients who had received 1 or more prior line of therapy. ${ }^{92}$ The results showed that the combination retained its greater efficacy in response rate, time to progression, and overall survival compared with dexamethasone alone in each subgroup of patients. In addition, lenalidomide plus dexamethasone appeared to be more active in patients with only 1 previous line of therapy than in those with more than 1 previous line. ${ }^{92}$ This supports its use at first relapse as opposed to later as salvage therapy.

Another pooled analysis from the same 2 studies examined the impact of prior thalidomide on outcomes. ${ }^{93}$ Although lenalidomide plus dexamethasone retained its superior efficacy compared with dexamethasone alone in both patients who had and those who had not received prior thalidomide, ${ }^{93}$ data indicated that activity of the combination was reduced in patients who had received prior thalidomide versus those who had not, in terms of response rate ( $53 \%$ vs. $63 \%$ ), complete response rate ( $8 \%$ vs. $18 \%$ ), and median time to progression ( 8.5 vs. 14.2 months). ${ }^{93}$ These findings suggest that for optimal efficacy, careful consideration of the sequencing of thalidomide versus lenalidomide is required, and in selected patients perhaps lenalidomide plus dexamethasone should be administered before a thalidomide-containing regimen.

Lenalidomide is also being investigated in combination with doxorubicin and dexamethasone, ${ }^{37}$ as well as with liposomal doxorubicin, dexamethasone, and vincristine (DVd-R) in the relapsed setting. ${ }^{38}$ Results have shown the DVd-R regimen to produce a $75 \%$ response rate (SWOG criteria), with 29\% complete or near complete responses and a median progression-free survival of 12 months. ${ }^{38}$

Compared with thalidomide, some aspects of the toxicity profile of lenalidomide appear reduced. Lenalidomide appears to produce a lower incidence of the characteristic dose-limiting toxicities of thalidomide such as somnolence, gastrointestinal side effects, and neuropathy. The most common grade 3 or higher toxicities are neutropenia and thrombocytopenia, which are manageable via dose reduction. ${ }^{36,91}$ However, as seen with thalidomide, lenalidomide in combination with dexamethasone is associated with an elevated risk of thromboembolic events, particularly in patients receiving concomitant erythropoietin. . $02,21,72,94,95$ As with thalidomide, antithrombotic prophylaxis is necessary to manage this risk. ${ }^{72,94-96}$ Moreover, higher rates of thromboses are seen with lenalidomide plus dexamethasone in patients who have been previously exposed to thalidomide, ${ }^{93}$ suggesting that this may be an important clinical consideration in deciding on sequencing therapies for a patient with relapsed MM.

\section{Other Novel Therapies}

A number of other novel therapies have shown antimyeloma activity, either alone or in combination, in both pre-clinical or clinical studies. These include arsenic trioxide, ${ }^{97-103}$ 2-methoxyestradiol, ${ }^{104-106}$ TRAIL/ Apo 2 ligand, ${ }^{10}$ cyclic depsipeptides, ${ }^{107}$ IL-1 receptor antagonists, ${ }^{108} \mathrm{Bcl}-2$ antisense ${ }^{109}$ and inhibitors of heat 
Novel Therapies in Relapsed Myeloma

shock proteins, ${ }^{110}$ farnesyl transferase, ${ }^{111} \mathrm{p} 38$ mitogenactivated pathway kinase, ${ }^{112}$ and histone deacetylase, ${ }^{113,114}$ as well as monoclonal antibodies. Arsenic trioxide is in relatively advanced stages of development, and researchers have evaluated combinations of arsenic trioxide plus ascorbic acid and melphalan or bortezomib in phase I and II studies. ${ }^{97,103}$ Indeed, other combination regimens incorporating other novel targeted agents with bortezomib, thalidomide, lenalidomide, or other commonly used agents represent a promising future direction for treating relapsed myeloma. Further investigation is needed to evaluate the efficacy and safety of these agents and regimens and the optimal combinations. The outlook for further improvements in response and chemical benefit is encouraging.

\section{Conclusions}

Novel therapeutic approaches involving bortezomib, thalidomide, and lenalidomide have shown substantial efficacy with manageable toxicities in a large number of clinical trials in relapsed MM, and their introduction and approval has significantly advanced the management of MM over the past decade. Their use is widening clinically, both alone and in combination regimens. Exploiting our increased understanding of the potential targets for MM therapy and their unique interplay will lead to further advances. The activity demonstrated in patients with disease previously refractory to conventional therapies is especially encouraging and has led to prolonged survival. Ongoing trials currently focus on combining these and other novel therapies with conventional antitumor agents and corticosteroids to identify regimens with the greatest efficacy and most manageable toxicity, with the goal of continued improvements in patient outcome.

\section{References}

1. Sirohi B, Powles R. Multiple myeloma. Lancet 2004;363:875-887.

2. Blade J, Samson D, Reece D, et al. Criteria for evaluating disease response and progression in patients with multiple myeloma treated by high-dose therapy and haemopoietic stem cell transplantation. Myeloma Subcommittee of the EBMT. European Group for Blood and Marrow Transplant. Br J Haematol 1998;102:1115-1123.

3. Durie BG, Harousseau JL, Miguel JS, et al. International uniform response criteria for multiple myeloma. Leukemia 2006;20: 1467-1473.

4. Hideshima T, Richardson P, Chauhan D, et al. The proteasome inhibitor PS-341 inhibits growth, induces apoptosis, and overcomes drug resistance in human multiple myeloma cells. Cancer Res 2001;61:3071-3076.

5. Hideshima T, Chauhan D, Schlossman R, et al. The role of tumor necrosis factor alpha in the pathophysiology of human multiple myeloma: therapeutic applications. Oncogene 2001;20: $\underline{4519-4527 .}$

6. Hideshima T, Chauhan D, Richardson P, et al. NF-kappa B as a therapeutic target in multiple myeloma. J Biol Chem 2002;277: 16639-16647.

7. Mitsiades N, Mitsiades CS, Poulaki V, et al. Biologic sequelae of nuclear factor-kappaB blockade in multiple myeloma: therapeutic applications. Blood 2002;99:4079-4086.

8. Hideshima T, Chauhan D, Hayashi T, et al. Proteasome inhibitor PS-341 abrogates IL-6 triggered signaling cascades via caspase-dependent downregulation of gp130 in multiple myeloma. Oncogene 2003;22:8386-8393.

9. Hideshima T, Mitsiades C, Akiyama M, et al. Molecular mechanisms mediating antimyeloma activity of proteasome inhibitor PS-341. Blood 2003;101:1530-1534.

10. Mitsiades CS, Treon SP, Mitsiades N, et al. TRAIL/Apo2L ligand selectively induces apoptosis and overcomes drug resistance in multiple myeloma: therapeutic applications. Blood 2001;98:795-804.

11. Mitsiades N, Mitsiades CS, Poulaki V, et al. Molecular sequelae of proteasome inhibition in human multiple myeloma cells. Proc Natl Acad Sci U S A 2002;99:14374-14379.

12. Mitsiades N, Mitsiades CS, Richardson PG, et al. The proteasome inhibitor PS-341 potentiates sensitivity of multiple myeloma cells to conventional chemotherapeutic agents: therapeutic applications. Blood 2003;101:2377-2380.

13. Orlowski RZ, Baldwin AS Jr. NF-kappaB as a therapeutic target in cancer. Trends Mol Med 2002;8:385-389.

14. Ma MH, Yang HH, Parker $K$, et al. The proteasome inhibitor PS341 markedly enhances sensitivity of multiple myeloma tumor cells to chemotherapeutic agents. Clin Cancer Res 2003;9:1136-1144.

15. Mitsiades N, Mitsiades CS, Poulaki V, et al. Apoptotic signaling induced by immunomodulatory thalidomide analogs in human multiple myeloma cells: therapeutic implications. Blood 2002;99: 4525-4530.

16. Jagannath $S$, Barlogie $B$, Berenson J, et al. A phase 2 study of two doses of bortezomib in relapsed or refractory myeloma. Br J Haematol 2004;127:165-172.

17. Richardson PG, Barlogie B, Berenson J, et al. A phase 2 study of bortezomib in relapsed, refractory myeloma. $\mathrm{N}$ Engl J Med 2003;348:2609-2617.

18. Richardson PG, Sonneveld P, Schuster MW, et al. Bortezomib or high-dose dexamethasone for relapsed multiple myeloma. N Engl J Med 2005;352:2487-2498.

19. Richardson P, Sonneveld P, Schuster M, et al. Bortezomib continues to demonstrate superior efficacy compared with high-dose dexamethasone in relapsed multiple myeloma: updated results of the APEX trial [abstract]. Blood 2005;106:715a. Abstract 2547.

20. Weber DM, Chen C, Niesvizky R, et al. Lenalidomide plus highdose dexamethasone provides improved survival compared to highdose dexamethasone alone for relapsed or refractory multiple myeloma: results of a North American phase III study (MM-009) [abstract]. J Clin Oncol 2006;24:427s. Abstract 7521.

21. Dimopoulos MA, Anagnostopoulos A, Prince M, et al. Lenalidomide (Revlimid) combination with dexamethasone (DEX) is more effective than DEX alone in patients with relapsed or refractory multiple myeloma and independent of the number of previous treatments [abstract]. Haematologica 2006;91(Suppl 1):181. Abstract 494. 
Richardson et al.

22. Hollmig K, Stover J, Talamo G, et al. Bortezomib (Velcade ${ }^{\mathrm{TM}}$ ) + Adriamycin ${ }^{\mathrm{TM}}+$ thalidomide + dexamethasone (VATD) as an effective regimen in patients with refractory or relapsed multiple myeloma (MM) [abstract]. Blood 2004;104:659a. Abstract 2399.

23. Leoni F, Casini C, Breschi C, et al. Low dose bortezomib, dexamethasone, thalidomide plus liposomal doxorubicin in relapsed and refractory myeloma [abstract]. Haematologica 2006;91(Suppl 1):281. Abstract 764 .

24. Zangari M, Barlogie B, Burns MJ, et al. Velcade (V)-thalidomide (T)-dexamethasone (D) for advanced and refractory multiple myeloma (MM): long-term follow-up of phase I-II trial UARK 2001-37: superior outcome in patients with normal cytogenetics and no prior T [abstract]. Blood 2005;106:717a. Abstract 2552.

25. Orlowski RZ, Voorhees PM, Garcia RA, et al. Phase 1 trial of the proteasome inhibitor bortezomib and pegylated liposomal doxorubicin in patients with advanced hematologic malignancies. Blood 2005;105:3058-3065.

26. Richardson P, Schlossman R, Munshi N, et al. A phase 1 trial of lenalidomide $\left(\right.$ REVLIMID $\left.^{\circledR}\right)$ with bortezomib $\left(V^{2} L C A D E^{\circledast}\right)$ in relapsed and refractory multiple myeloma [abstract]. Blood 2005;106:110a. Abstract 365.

27. Padmanabhan S, Miller K, Musiel L, et al. Bortezomib (Velcade) in combination with liposomal doxorubicin (Doxil) and thalidomide is an active salvage regimen in patients with relpase or refractory multiple myeloma: final results of a phase II trial [abstract]. Haematologica 2006;91(Suppl 1):277. Abstract 752.

28. Palumbo A, Ambrosini MT, Benevolo G, et al. Bortezomib, melphalan, prednisone and thalidomide for relapsed multiple myeloma. Blood 2006; e-pub ahead of print.

29. Terpos E, Anagnostopoulos A, Kastritis E, et al. The combination of bortezomib, melphalan, dexamethasone and intermittent thalidomide (VMTD) is an effective regimen for relapsed/refractory myeloma and reduces serum levels of RANKL, MIP- 1 alpha and angiogenic cytokines [abstract]. Haematologica 2006;91(Suppl 1):84. Abstract 223.

30. Barlogie B, Desikan R, Eddlemon P, et al. Extended survival in advanced and refractory multiple myeloma after single-agent thalidomide: identification of prognostic factors in a phase 2 study of 169 patients. Blood 2001;98:492-494.

31. Palumbo A, Bertola A, Falco P, et al. Efficacy of low-dose thalidomide and dexamethasone as first salvage regimen in multiple myeloma. Hematol J 2004;5:318-324.

32. Dimopoulos MA, Hamilos G, Zomas A, et al. Pulsed cyclophosphamide, thalidomide and dexamethasone: an oral regimen for previously treated patients with multiple myeloma. Hematol J 2004;5:112-117.

33. Offidani M, Corvatta L, Marconi M, et al. Low-dose thalidomide with pegylated liposomal doxorubicin and high-dose dexamethasone for relapsed/refractory multiple myeloma: a prospective, multicenter, phase II study. Haematologica 2006;91:133-136.

34. Hussein MA, Baz R, Srkalovic G, et al. Phase 2 study of pegylated liposomal doxorubicin, vincristine, decreased-frequency dexamethasone, and thalidomide in newly diagnosed and relapsedrefractory multiple myeloma. Mayo Clin Proc 2006;81:889-895.

35. Palumbo A, Avonto I, Bruno B, et al. Intravenous melphalan, thalidomide and prednisone in refractory and relapsed multiple myeloma. Eur J Haematol 2006;76:273-277.

36. Richardson P, Jagannath $\mathrm{S}$, Hussein M, et al. A multicenter, single-arm, open-label study to evaluate the efficacy and safety of single-agent lenalidomide in patients with relapsed and refractory multiple myeloma; preliminary results [abstract]. Blood 2005; 106:449a. Abstract 1565.

37. Gerecke C, Knop S, Topp MS, et al. A multicenter phase I/II trial evaluating the safety and efficacy of lenalidomide [Revlimid ${ }^{\circledR}$ CC5013] in combination with doxorubicin and dexamethasone (RAD) in patients with relapsed or refractory multiple myeloma [abstract]. Blood 2005;106:367b. Abstract 5136.

38. Baz R, Walker E, Karam MA, et al. Lenalidomide and pegylated liposomal doxorubicin-based chemotherapy for relapsed or refractory multiple myeloma: safety and efficacy. Ann Oncol 2006;17: 1766-1771.

39. Sonneveld P, Richardson PG, Schuster MW, et al. Bortezomib at first relapse is superior to high-dose dexamethasone and more effective than when given later in relapsed multiple myeloma [abstract]. Haematologica 2005;90:146-147. Abstract P140.721.

40. Vogl DT, Stadtmauer EA, Richardson PG, et al. Impact of prior autologous stem cell transplant (ASCT) in patients receiving bortezomib or dexamethasone for relapsed/refractory multiple myeloma in the APEX trial [abstract]. J Clin Oncol 2006;24(Suppl 1):433s. Abstract 7546

41. Richardson PG, Sonneveld P, Schuster MW, et al. Safety and efficacy of bortezomib in high-risk and elderly patients with relapsed myeloma [abstract]. J Clin Oncol 2005;23(Suppl 1):568s. Abstract 6533.

42. Knudsen LM, Hippe E, Hjorth M, et al. Renal function in newly diagnosed multiple myeloma-a demographic study of 1353 patients. The Nordic Myeloma Study Group. Eur J Haematol 1994;53:207-212.

43. Knudsen LM, Hjorth M, Hippe E. Renal failure in multiple myeloma: reversibility and impact on the prognosis. Nordic Myeloma Study Group. Eur J Haematol 2000;65:175-181.

44. Chanan-Khan AA, Kaufman JL, Mehta J, et al. Activity and safety of bortezomib in multiple myeloma patients with advanced renal failure: a multicenter retrospective study. Blood 2006; e-pub ahead of print.

45. Jagannath S, Barlogie B, Berenson JR, et al. Bortezomib in recurrent and/or refractory multiple myeloma. Initial clinical experience in patients with impared renal function. Cancer 2005;103: 1195-1200.

46. Mohrbacher A, Levine AM. Reversal of advanced renal dysfunction on bortezomib treatment in multiple myeloma patients [abstract]. J Clin Oncol 2005;23(Suppl 1):612s. Abstract 6714.

47. Mulkerin D, Remick S, Ramanathan R, et al. A dose-escalating and pharmacologic study of bortezomib in adult cancer patients with impaired renal function [abstract]. J Clin Oncol 2006;24(Suppl 1):87s. Abstract 2032.

48. Nozza A, Siracusano L, Armando S. Bortezomib-dexamethasone combination in a patient with refractory multiple myeloma and impaired renal function. Clin Ther 2006;28:953-959.

49. Lonial S, Richardson P, Sonneveld P, et al. Hematologic profiles in the phase 3 APEX trial [abstract]. Blood 2005;106:970a. Abstract 3474.

50. Lonial S, Waller EK, Richardson PG, et al. Risk factors and kinetics of thrombocytopenia associated with bortezomib for relapsed, refractory multiple myeloma. Blood 2005;106:3777-3784.

51. Richardson PG, Briemberg H, Jagannath $S$, et al. Frequency, characteristics, and reversibility of peripheral neuropathy during treatment of advanced multiple myeloma with bortezomib. J Clin Oncol 2006;24:3113-3120.

52. San Miguel JF, Richardson P, Sonneveld P, et al. Frequency, characteristics, and reversibility of peripheral neuropathy $(\mathrm{PN})$ in the APEX trial [abstract]. Blood 2005;106:111a. Abstract 366. 
53. Anderson KC. Lenalidomide and thalidomide: mechanisms of action—similarities and differences. Semin Hematol 2005;42: S3-8.

54. Richardson $P$, Anderson K. Immunomodulatory analogs of thalidomide: an emerging new therapy in myeloma. J Clin Oncol 2004;22: 3212-3214.

55. Hideshima T, Chauhan D, Shima Y, et al. Thalidomide and its analogs overcome drug resistance of human multiple myeloma cells to conventional therapy. Blood 2000;96:2943-2950.

56. D'Amato RJ, Loughnan MS, Flynn E, Folkman J. Thalidomide is an inhibitor of angiogenesis. Proc Natl Acad Sci USA 1994;91: 4082-4085.

57. Geitz H, Handt $\mathrm{S}$, Zwingenberger $\mathrm{K}$. Thalidomide selectively modulates the density of cell surface molecules involved in the adhesion cascade. Immunopharmacology 1996;31:213-221.

58. Haslett PA, Corral LG, Albert M, Kaplan G. Thalidomide costimulates primary human $\mathrm{T}$ lymphocytes, preferentially inducing proliferation, cytokine production, and cytotoxic responses in the CD8+ subset. J Exp Med 1998;187:1885-1892.

59. Rajkumar SV, Fonseca R, Dispenzieri A, et al. Thalidomide in the treatment of relapsed multiple myeloma. Mayo Clin Proc 2000;75: 897-901.

60. Schey SA, Cavenagh J, Johnson R, et al. An UK myeloma forum phase II study of thalidomide; long term follow-up and recommendations for treatment. Leuk Res 2003;27:909-914.

61. Singhal S, Mehta J, Desikan R, et al. Antitumor activity of thalidomide in refractory multiple myeloma. N Engl J Med 1999;341:1565-1571.

62. Yakoub-Agha I, Attal M, Dumontet $\mathrm{C}$, et al. Thalidomide in patients with advanced multiple myeloma: a study of 83 patients—-report of the Intergroupe Francophone du Myelome (IFM). Hematol J 2002;3:185-192.

63. Glasmacher A, Hahn C, Hoffmann F, et al. A systematic review of phase-II trials of thalidomide monotherapy in patients with relapsed or refractory multiple myeloma. Br J Haematol 2006;132: 584-593.

64. Juliusson G, Celsing F, Turesson I, et al. Frequent good partial remissions from thalidomide including best response ever in patients with advanced refractory and relapsed myeloma. Br J Haematol 2000;109:89-96.

65. Richardson P, Schlossman R, Jagannath S, et al. Thalidomide for patients with relapsed multiple myeloma after high-dose chemotherapy and stem cell transplantation: results of an open-label multicenter phase 2 study of efficacy, toxicity, and biological activity. Mayo Clin Proc 2004;79:875-882.

66. Matthews SJ, McCoy C. Thalidomide: a review of approved and investigational uses. Clin Ther 2003;25:342-395.

67. Candelaria D, Armijo B, Montgomery R, et al. Thalidomide increases thrombin generation in multiple myeloma patients [abstract]. J Clin Oncol 2006;24(Suppl 1):447s. Abstract 7602.

68. Naina HVK, Lacy MQ, Dispenzieri A, et al. Incidence and clinical course of peripheral neuropathy in patients receiving thalidomide for the treatment of multiple myeloma [abstract]. Blood 2005;106:970a. Abstract 3475.

69. Mileshkin L, Stark R, Day B, et al. Development of neuropathy in patients with myeloma treated with thalidomide: patterns of occurrence and the role of electrophysiologic monitoring. J Clin Oncol 2006;24:4507-4514.

70. Yakoub-Agha I, Doyen C, Hulin C, et al. A multicenter prospective randomized study testing non-inferiority of thalidomide 100 $\mathrm{mg} /$ day as compared with $400 \mathrm{mg} /$ day in patients with refractory/relapsed multiple myeloma: Results of the final analysis of the
IFM 01-02 study [abstract]. J Clin Oncol 2006;24(Suppl 1):427s. Abstract 7520

71. Celgene Corporation. THALOMID (thalidomide) Product Information. Summit, NJ; 2006. Available at www.celgene.com. Accessed February 9, 2007.

72. Hussein MA. Thromboembolism risk reduction in multiple myeloma patients treated with immunomodulatory drug combinations. Thromb Haemost 2006;95:924-930.

73. Rajkumar SV, Blood E, Vesole D, et al. Phase III clinical trial of thalidomide plus dexamethasone compared with dexamethasone alone in newly diagnosed multiple myeloma: a clinical trial coordinated by the Eastern Cooperative Oncology Group. J Clin Oncol 2006;24:431-436.

74. Rajkumar SV, Hussein M, Catalano J, et al. A multicenter, randomized, double-blind, placebo-controlled trial of thalidomide plus dexamethasone versus dexamethasone alone as initial therapy for newly diagnosed multiple myeloma [abstract]. J Clin Oncol 2006; 24(Suppl 1):426s. Abstract 7517.

75. Baz R, Kottke-Marchant K, Srkalovic G, et al. The role of aspirin in the prevention of thrombotic complications of thalidomide and anthracycline-based chemotherapy for multiple myeloma. Mayo Clin Proc 2005;80:1568-1574.

76. Rajkumar SV. Thalidomide therapy and deep venous thrombosis in multiple myeloma. Mayo Clin Proc 2005;80:1549-1551.

77. Hatjiharissi E, Terpos E, Papaioannou M, et al. The combination of intermediate doses of thalidomide and dexamethasone reduces bone marrow micro-vessel density but not serum levels of angiogenic cytokines in patients with refractory/relapsed multiple myeloma. Hematol Oncol 2004;22:159-168.

78. Palumbo A, Giaccone L, Bertola A, et al. Low-dose thalidomide plus dexamethasone is an effective salvage therapy for advanced myeloma. Haematologica 2001;86:399-403.

79. Palumbo A, Falco P, Ambrosini MT, et al. Thalidomide plus dexamethasone is an effective salvage regimen for myeloma patients relapsing after autologous transplant. Eur J Haematol 2005;75: 391-395.

80. Terpos E, Mihou D, Szydlo R, et al. The combination of intermediate doses of thalidomide with dexamethasone is an effective treatment for patients with refractory/relapsed multiple myeloma and normalizes abnormal bone remodeling, through the reduction of sRANKL/osteoprotegerin ratio. Leukemia 2005;19: 1969-1976.

81. Glasmacher A, Hahn C, Hoffmann F, et al. Thalidomide in relapsed or refractory patients with multiple myeloma: monotherapy or combination therapy? A report from systematic reviews [abstract]. Blood 2005;106:364b. Abstract 5125.

82. Garcia-Sanz R, Gonzalez-Porras JR, Hernandez JM, et al. The oral combination of thalidomide, cyclophosphamide and dexamethasone (ThaCyDex) is effective in relapsed/refractory multiple myeloma. Leukemia 2004;18:856-863.

83. Kyriakou C, Thomson K, D'Sa S, et al. Low-dose thalidomide in combination with oral weekly cyclophosphamide and pulsed dexamethasone is a well tolerated and effective regimen in patients with relapsed and refractory multiple myeloma. Br J Haematol 2005;129:763-770.

84. Sidra G, Williams CD, Russell NH, et al. Combination chemotherapy with cyclophosphamide, thalidomide and dexamethasone for patients with refractory, newly diagnosed or relapsed myeloma. Haematologica 2006;91:862-863.

85. Massimo MO, Bringhen S, Corvatta L, et al. Thalidomide-dexamethasone vs thalidomide-dexamethasone-pegylated liposomal 
Richardson et al.

doxorubicin: a case-matched study in patients with advanced multiple myeloma [abstract]. Haematologica 2006;91(Suppl 1):89. Abstract 236.

86. Davies FE, Raje N, Hideshima T, et al. Thalidomide and immunomodulatory derivatives augment natural killer cell cytoxicity in multiple myeloma. Blood 2001;98:210-216.

87. Gupta D, Treon SP, Shima Y, et al. Adherence of multiple myeloma cells to bone marrow stromal cells upregulates vascular endothelial growth factor secretion: therapeutic applications. Leukemia 2001;15:1950-1961.

88. Lentzsch S, LeBlanc R, Podar K, et al. Immunomodulatory analogs of thalidomide inhibit growth of Hs Sultan cells and angiogenesis in vivo. Leukemia 2003;17:41-44.

89. Zangari M, Elice F, Tricot G. Immunomodulatory drugs in multiple myeloma. Expert Opin Investig Drugs 2005;14:1411-1418.

90. Richardson PG, Blood E, Mitsiades C, et al. A randomized phase 2 study of lenalidomide therapy for patients with relapsed or relapsed and refractory multiple myeloma. Blood 2006;108:3458-3464.

91. Richardson PG, Schlossman RL, Weller E, et al. Immunomodulatory drug CC-5013 overcomes drug resistance and is well tolerated in patients with relapsed multiple myeloma. Blood 2002;100: 3063-3067.

92. Stadtmauer EA, Weber D, Dimopoulos MA, et al. Lenalidomide (Len) in combination with dexamethasone (Dex) is more effective than Dex alone at first relapse and provides better outcomes when used early rather than as later salvage therapy in relapsed multiple myeloma (MM) [abstract]. J Clin Oncol 2006;24(Suppl 1):446s. Abstract 7600.

93. Wang M, Knight R, Dimopoulos M, et al. Comparison of lenalidomide in combination with dexamethasone to dexamethasone alone in patients who have received prior thalidomide in relpased or refractory multiple myeloma [abstract]. J Clin Oncol 2006;24(Suppl 1):427s. Abstract 7522.

94. Knight R, DeLap RJ, Zeldis JB. Lenalidomide and venous thrombosis in multiple myeloma. N Engl J Med 2006;354:2079-2080.

95. Niesvizky R, Spencer A, Wang M, et al. Increased risk of thrombosis with lenalidomide in combination with dexamethasone and erythropoietin [abstract]. J Clin Oncol 2006;24(Suppl 1):423s. Abstract 7506.

96. Zonder JA, Durie BGM, McCoy J, et al. High incidence of thrombotic events observed in patients receiving lenalidomide $(\mathrm{L})+$ dexamethasone (D) (LD) as first-line therapy for multiple myeloma (MM) without aspirin (ASA) prophylaxis [abstract]. Blood 2005; 106:964a. Abstract 3455.

97. Berenson JR, Matous JV, Ferretti D, et al. A phase I/II study of arsenic trioxide, bortezomib and ascorbic acid in relapsed or refactory multiple myeloma [abstract]. J Clin Oncol 2006;24 (Suppl 1):449s.

98. Hussein MA, Saleh M, Ravandi F, et al. Phase 2 study of arsenic trioxide in patients with relapsed or refractory multiple myeloma. Br J Haematol 2004;125:470-476.

99. Munshi NC, Tricot G, Desikan R, et al. Clinical activity of arsenic trioxide for the treatment of multiple myeloma. Leukemia 2002; 16: 1835-1837.

100. $\overline{\text { Qazilbash }}$ MH, Davis MS, Ana A, et al. Arsenic trioxide with ascorbic acid and high-dose melphalan: a new preparative regimen for autologous hematopoietic stem cell transplantation for multiple myeloma [abstract]. Blood 2005;106:338a. Abstract 1159.

101. Rousselot P, Larghero J, Arnulf B, et al. A clinical and pharmacological study of arsenic trioxide in advanced multiple myeloma patients. Leukemia 2004;18:1518-1521.

102. Wu K, van Droogenbroeck J, Beksac M, et al. Treatment with arsenic trioxide, ascorbic acid and dexamethasone in advanced myeloma patients: preliminary findings of a multicenter, phase II study [abstract]. Blood 2005;106:367a. Abstract 5135.

103. Berenson J, Boccia R, Siegel D, et al. A multicenter phase II study of combination treatment with melphalan, arsenic trioxide and vitamin $\mathrm{C}$ (MAC) for patients with relapsed or refractory multiple myeloma [abstract]. Blood 2005;106:720a. Abstract 2564.

104. Chauhan D, Catley L, Hideshima T, et al. 2-Methoxyestradiol overcomes drug resistance in multiple myeloma cells. Blood 2002;100:2187-2194.

105. Chauhan D, Li G, Auclair D, et al. 2-Methoxyestardiol and bortezomib/proteasome-inhibitor overcome dexamethasone-resistance in multiple myeloma cells by modulating Heat Shock Protein-27. Apoptosis 2004;9:149-155.

106. Dingli D, Timm M, Russell SJ, et al. Promising preclinical activity of 2-methoxyestradiol in multiple myeloma. Clin Cancer Res 2002;8:3948-3954.

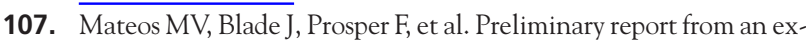
ploratory phase II trial with plitidepsin (Aplidin $(\mathrm{R}))$ in patients with refractory/relapsed multiple myeloma [abstract]. Blood 2005; 106:722a. Abstract 2569.

108. Lust JA, Lacy MQ, Zeldenrust SR, et al. In smoldering/indolent (SMM/IMM) myeloma patients treated with interleukin-1 receptor antagonist (IL-1Ra), responders demonstrate a significantly increased time to progression (TTP) and a decreased C-reactive protein (CRP) compared with nonresponders [abstract]. Blood 2005;106:721a. Abstract 2567.

109. van de Donk NW, de WO, Veth G, et al. G3139, a Bcl-2 antisense oligodeoxynucleotide, induces clinical responses in VAD refractory myeloma. Leukemia 2004;18:1078-1084.

110. Richardson PG, Chanan-Khan AA, Alsina M, et al. Safety and activity of KOS-953 in patients with relapsed refractory multiple myeloma (MM): interim results of a phase 1 trial [abstract]. Blood 2005;106:109a. Abstract 361.

111. Alsina $\mathrm{M}$, Fonseca R, Wilson EF, et al. Farnesyltransferase inhibitor tipifarnib is well tolerated, induces stabilization of disease, and inhibits farnesylation and oncogenic/tumor survival pathways in patients with advanced multiple myeloma. Blood 2004;103: 3271-3277.

112. Nguyen AN, Young GO, Quon D, et al. SCIO-469, a potent and selective inhibitor of the $\mathrm{p} 38$ a MAPK, inhibits TNFa-induced adhesion of multiple myeloma cells to bone marrow stromal cells via downregulation of chemokines CXCL10/IP-10 and CCL8 [abstract]. Blood 2005;106:450a. Abstract 1569.

113. Catley L, Weisberg E, Tai YT, et al. NVP-LAQ824 is a potent novel histone deacetylase inhibitor with significant activity against multiple myeloma. Blood 2003;102:2615-2622.

114. Mitsiades CS, Mitsiades NS, McMullan CJ, et al. Transcriptional signature of histone deacetylase inhibition in multiple myeloma: biological and clinical implications. Proc Natl Acad Sci USA 2004;101: 540-545. 